\title{
Sleep Disorders in Neuromuscular \\ Diseases: Adaption in Care Provision During the COVID-19 Pandemic
}

\author{
Katherine E Cox-Flaherty ${ }^{1,2}$ and Eric J Gartman ${ }^{1}$ \\ 1. The Warren Alpert School of Medicine of Brown University, Providence, RI, USA; Providence VA Medical Center, Providence RI, USA; 2. \\ Lifespan Hospital System, Providence, RI, USA
}

\section{Keywords}

Sleep disorders, neuromuscular disease, COVID-19, pandemic, adaptations.

Disclosure: Katherine E Cox-Flaherty and Eric J Gartman have no financial or non-financial relationships or activities to declare in relation to this article.

Review Process: Double-blind peer review.

Authorship: The named authors meet the International Committee of Medical Journal Editors (ICMJE) criteria

for authorship of this manuscript, take responsibility for the integrity of the work as a whole, and have given final approval for the version to be published.

Received: 29 September 2020

Accepted: 10 March 2021

Published Online: 25 June 2021

Citation: touchREVIEWS in Respiratory \& Pulmonary Diseases. 2021;6(1):5-7

Corresponding author: Katherine E Cox-Flaherty, Division of Pulmonary, Critical Care, and Sleep Medicine, Rhode Island Hospital, 593 Eddy Street, POB Suite 224, Providence, RI 02903, USA. E: katherine_cox@brown.edu

Support: No funding was received for

the publication of this article.
Sleep disturbances are exceedingly common and potentially morbid in patients with neuromuscular diseases (NMD). ${ }^{1}$ A constellation of physiologic changes occur in patients with NMDs which, in concert with normal physiologic changes during sleep, lead to decreased nocturnal ventilation that often precedes daytime hypoventilation. The standard manner in which we diagnose and treat sleep disorders in this patient population has become exceedingly challenging in the COVID-19 era. In this editorial, we aim to review healthcare delivery barriers that have been presented by the pandemic and how we can adapt our practice to maximize the care of NMD patients.

\section{Physiology during sleep}

A basic understanding of physiologic alterations during sleep in NMD patients is required to direct appropriate testing and treatment. A detailed review of the pathophysiology of sleepdisordered breathing in neuromuscular diseases has been described previously. ${ }^{2}$ While most clinicians recognize that patients with advanced NMD may develop issues with hypoventilation - at first, during sleep then, as their disease progresses, during wakefulness - it is important to appreciate that the spectrum of NMDs is highly variable and most patients do not develop clinically overt chronic ventilatory failure. NMDs span a wide range of conditions - from diaphragm disorders, congenital musculoskeletal abnormalities, relapsing/remitting neurologic conditions, to progressive neuromuscular disorders - and many are associated with sleep-disordered breathing that will go unnoticed if not formally evaluated. In addition to common sleep-disordered breathing issues, depending on the disease, NMD patients have additional physiologic alterations that lead to additional sleep abnormalities. For example, nocturnal hypoventilation occurs due to muscle atonia associated with rapid eye movement (REM), respiratory muscle weakness and, particularly, diaphragmatic weakness. ${ }^{2}$ Patients with bulbar muscle involvement may have additional airway issues during sleep. Central apnoea events are also common in this patient population for a variety of reasons depending upon the underlying pathology. Finally, paroxysmal limb movements are also common in several NMD conditions and should be screened for in these patients. ${ }^{3}$

\section{Evaluation of sleep disorders in neuromuscular disease in the COVID-19 era}

As stated above, patients with 'neuromuscular disease' are quite varied in their presentation and pathophysiology, and the workup (and subsequent treatment) of each patient needs to be individualized. For example, for patients with advanced progressive NMDs who clearly have chronic respiratory failure, empiric treatment with non-invasive ventilation is indicated and further testing likely is not needed. However, most patients do not always present with a known disorder or clear clinical picture, and a thorough evaluation is recommended (Table 1). 
Table 1: Pros and cons of the conventional methods for assessing sleep disturbances when applied to patients with neuromuscular diseases

\begin{tabular}{|l|l|l|}
\hline & Pro & Con \\
\hline Overnight oximetry & Convenient and easily accessible & Short or mild episodes of hypoventilation may be missed \\
\hline Home sleep assessment test & Convenient and easily accessible & $\begin{array}{l}\text { Will not reliably detect hypoventilation or central events } \\
\text { Unsupervised, integrity of the test may be compromised by external factors }\end{array}$ \\
\hline Attended polysomnogram & $\begin{array}{l}\text { Gold standard for sleep evaluation and treatment } \\
\text { titration for obstructive sleep apnoea }\end{array}$ & $\begin{array}{l}\text { Can be difficult to orchestrate; limited to closed laboratories. } \\
\text { Titration studies may be prohibited }\end{array}$ \\
\hline
\end{tabular}

Infection control efforts due to the COVID-19 pandemic have varied widely and evolved over time, but suffice to say that they have hindered our ability to evaluate patients in the standard manner. Our first step in the evaluation remains straightforward, assuming access to care has been maintained. Through taking the patient's history, we must ascertain the presence of morning headaches, daytime fatigue, generalized fatigue, excessive sleep time with nocturnal awakenings, snoring, witnessed apnoeas and shortness of breath with positional changes (due to changes in chest wall compliance). If there is any suspicion of an underlying sleep or ventilatory disorder, the evaluation should include checking for an elevated serum bicarbonate and elevated carbon dioxide level, which may be suggestive of chronic hypoventilation (though it may be absent without daytime hypoventilation). ${ }^{4}$

The next steps can include pulmonary function testing (PFT), specifically manoeuvres to evaluate respiratory muscle strength (e.g. maximal inspiratory pressure [MIP], maximal expiratory pressure [MEP] upright and supine spirometry); however, many pulmonary function laboratories remain prone to closure or have significant limitations on testing. Pretesting for SARS-COV-2 is often required, which can be an insurmountable barrier given the mobility constraints of many NMD patients and the excessive wait times for test results. Some PFT laboratories have decided not to require SARS-COV-2 screening prior to testing, and instead have adapted their laboratories as full negative pressure rooms or outfitted the technicians in full protective gear for the duration of testing. Regardless of strategy, most PFT laboratories are operating at a reduced capacity due to infection control constraints.

Attended polysomnograms (PSG) including transcutaneous $\mathrm{CO}_{2}$ monitoring are the gold standard for assessment of sleep disturbances given their ability to create a comprehensive physiologic analysis of a patient's sleep. This includes not only documenting obstructive and central events but also profiling subtle increases in $\mathrm{PCO}_{2}$ and decreases in $\mathrm{O}_{2}$ during REM sleep; patients with NMD are at increased risk for all of these. However, performing in-lab PSGs has become challenging during the COVID pandemic. Orchestrating full PSGs can be difficult in NMD patients with mobility issues at baseline, and currently with sleep laboratory closures and limitations it could prove impossible. Most laboratories that have reopened are requiring SARS-COV-2 pre-testing, which presents similar challenges as described above.

Due to these issues, the concept of home testing becomes more appealing. However, it is important to remember that home studies are limited and only useful in patients with a high pre-test probability for uncomplicated moderate to severe obstructive sleep apnoea without additional sleep disorders. ${ }^{5}$ As such, home studies are not well suited for NMD patients as they are not designed to detect hypoventilation or central sleep apnoeas, and more advanced monitoring cannot be performed. ${ }^{5}$ In the current climate, a home study would only be appropriate if the physician suspects concomitant obstructive sleep apnoea, but would not be able to diagnose hypoventilation. Based upon symptoms or the severity of neuromuscular disease, it would be reasonable to start with nocturnal oximetry testing, which would detect severe nocturnal hypoventilation. If there is any suggestion of symptoms consistent with nocturnal hypoventilation, sleep-disordered breathing or potentially hypoventilation-driven oxygen desaturation on nocturnal oximetry, empiric treatment should be considered.

\section{Treatment}

The prescription for sleep-related ventilatory disorders in NMD patients is directed most effectively by the comprehensive physiologic profile provided by in-laboratory monitoring and titration. While some advanced NMD patients may be managed empirically with non-invasive ventilation, most NMD patients will not have overt chronic respiratory failure, and nocturnal assessment and treatment need to be tailored and adapted to an individual's needs. This assessment allows for the evaluation of comorbid obstructive and central apnoeas, as well as the titration of expiratory airway pressures that will ensure airway patency and assure effective ventilation. Further, the formal interaction with trained respiratory therapists in this setting offers a unique opportunity for acclimation to the initiation of treatment.

Unfortunately, for the reasons stated above, such testing and personal interaction have become difficult to reliably obtain for many NMD patients. Depending on the clinical picture and the ability to obtain some elements of the evaluation, a provider may choose to implement therapy. Continuous positive airway pressure (CPAP) is generally not appropriate for use in patients with evidence of central apnoeas or hypoventilation, although some conditions may only affect the upper airway and CPAP would be indicated. For similar reasons, simple supplemental oxygen is not a sufficient therapy. Non-invasive positive pressure ventilation (NPPV) generally is the most appropriate therapy and can improve survival and quality of life. ${ }^{6-8} \mathrm{~A}$ bi-level device with a backup rate is the most commonly employed NPPV mode and can be used on an intermittent basis or eventually provide full ventilatory support. Newer modes of NPPV have been developed that theoretically improve upon the standard bi-level with a backup rate such as average volume-assured pressure support and intelligent volume-assured pressure support. While they have not demonstrated superiority with regard to functionality or survival in large studies over standard NPPV, mechanistically they are well suited to patients with NMD as they are designed to deliver a stable level of ventilation despite variation in respiratory efforts in different sleep stages. ${ }^{2}$ Medicare is currently covering positive airway pressure (PAP) devices based upon the clinician's assessment of the patient without requiring a formal diagnosis with in-lab PSG or home sleep apnoea testing. Of note, it remains unclear what follow-up testing may be required after this is pandemic is over.

Given the empiric nature of this treatment strategy, frequent communication with the patient and remote interrogation of the device 
are suggested to ensure efficacy and safety. Home visits conducted by the respiratory therapists employed by the home care equipment supplier or specialized care teams (if available) can be invaluable in the current environment to assist in the initiation of and acclimation to NPPV, given the inconsistent availability of in-laboratory or in-person clinic assessments and follow-up. Titrating therapy remains challenging. Home transcutaneous capnometry $\left(\mathrm{TCCO}_{2}\right)$ and ventilator-integrated end-tidal $\mathrm{CO}_{2}$ monitoring are options to estimate overnight $\mathrm{PCO}_{2}$ and ultimately ventilation. Both methods of home monitoring are valuable in that they may detect subtle transient episodes of hypoventilation not detected with intermittent blood gas assessment. It is notable that home $\mathrm{TCCO}_{2}$ monitoring remains expensive and somewhat impractical; however, in small studies, ventilator-integrated end-tidal $\mathrm{CO}_{2}$ monitoring seems to correlate well with both $\mathrm{TCCO}_{2}$ values and morning $\mathrm{PCO}_{2}$ values, and has the advantage of being relatively low cost. ${ }^{9}$ The existence of concomitant underlying parenchymal pulmonary disease would impact the accuracy of end tidal $\mathrm{CO}_{2}$ monitoring. The accuracy of both of these techniques is limited and should not completely replace blood gases. An additional challenge includes management of home PAP therapy in patients with potential COVID-19 infection given that NPPV is an aerosol-generating device and could, theoretically, disperse infective aerosols and spread to others. The risks remain unknown, and best clinical judgement should be applied. Theoretically, the use of nonvented masks with exhalation valves with viral filters and/or the use of closed-circuit ventilators could be employed to reduce transmission risk in these circumstances; however, it is unclear if these are reliably effective or would be logistically/financially feasible on a large scale. Further, despite these adaptations, significant uncertainty still exists, and even with these precautions in place maximum personal protective equipment should be employed (e.g. many hospitals currently place anyone on PAP therapy on aerosol precautions).

\section{Access to care}

The COVID pandemic has radically transformed clinical care delivery worldwide. In an effort to limit exposure to patients and medical staff, telemedicine has been widely adopted. It has the advantage of providing rapid and easy access to providers. Studies across several specialties suggest non-inferiority of virtual evaluations compared with in-person evaluations in terms of patient and caregiver satisfaction. ${ }^{10}$ The majority of NMDs progress slowly and are primarily managed on an outpatient basis, making them particularly appropriate for management via telemedicine. ${ }^{11}$ Additionally, patients with NMDs and respiratory failure often have challenges mobilizing personnel and equipment to attend clinic visits. Finally, there are fairly few providers who specialize in patients with NMDs and chronic respiratory failure, thus telemedicine may improve overall access to specialists. One randomized trial using telehealth in patients with amyotrophic lateral sclerosis demonstrated a reduced rate of hospitalizations. ${ }^{12}$ Long-term heavy reliance on telehealth has practical barriers to widespread implementation, including issues related to both commercial- and government-based insurance reimbursement and credentialing, as well as furthering inequities to patients without access to requisite electronic devices. ${ }^{13}$
1. Guilleminault C, Shergill RP. Sleep-disordered breathing in neuromuscular disease. Curr Treat Options Neurol. 2002:4:107-12.

2. Gartman E Sleep disorders in neuromuscular diseases. US Respir Pulm Dis. 2018:3:27-32.

3. Fereshtehnejad SM, Rahmani A, Shafieesabet M, et al. Prevalence and associated comorbidities of restless legs syndrome (RLS): Data from a large population-based doorto-door survey on 19176 adults in Tehran, Iran. Plos One. 2017; 12:e0172593

4. Bersanini C, Khirani S, Ramirez A, et al. Nocturnal hypoxaemia and hypercapnia in children with neuromuscular disorders. Eur Respir J. 2012;39:1206-12.

5. Kapur VK, Auckley DH, Chowdhuri S, et al. Clinical practice guideline for diagnostic testing for adult obstructive sleep apnea: An American Academy of Sleep Medicine Clinica Practice Guideline. J Clin Sleep Med. 2017;13:479-504.

6. Bourke SC, Tomlinson M, Williams TL, et al. Effects of noninvasive ventilation on survival and quality of life in patients with amyotrophic lateral sclerosis: a randomised controlled trial. Lancet Neurol. 2006:5:140-7.

7. Bach JR, Martinez D. Duchenne muscular dystrophy: continuous noninvasive ventilatory support prolongs survival. Respir Care 2011;56:744-50.

8. Berlowitz DJ, Howard ME, Fiore JF Jr, et al. Identifying who will benefit from non-invasive ventilation in amyotrophic lateral sclerosis/motor neurone disease in a clinical cohort. J Neurol Neurosurg Psychiatr. 2016;87:280-6

9. Orlikowski D, Prigent $H$, Ambrosi $X$, et al. Comparison of ventilator-integrated end-tidal $\mathrm{CO} 2$ and transcutaneous $\mathrm{CO} 2$ monitoring in home-ventilated neuromuscular patients. Respir Med. 2016;117:7-13.

10. Hatcher-Martin JM, Adams JL, Anderson ER, et al. Telemedicine in neurology: Telemedicine Work Group of the American Academy of Neurology update. Neurology. 2020;94:30-8.

11. Garibaldi M, Siciliano $G$, Antonini G. Telemedicine for neuromuscular disorders during the COVID-19 outbreak. J Neurol. 2021;268:1-4.

12. Hobson EV, Baird WO, Cooper CL, et al. Using technology to improve access to specialist care in amyotrophic latera sclerosis: A systematic review. Amyotroph Lateral Scler Frontotemporal Degener. 2016;17:313-24.

13. Hollander JE, Carr BG. Virtually Perfect? Telemedicine for Covid-19. NEJM. 2020;382:1679-81. 